\title{
Fast patterning microstructures using inkjet printing conformal masks
}

\author{
C.-H. Lin $\cdot$ H. Yang $\cdot$ F.-Y. Chang $\cdot$ \\ S.-H. Chang $\cdot$ M.-T. Yen
}

Published online: 8 October 2008

(c) Springer-Verlag 2008

Erratum to: Microsyst Technol (2008) 14:1263-1267

DOI 10.1007/s00542-007-0521-z

Unfortunately, an error occured in the author affiliations.

The correct affiliations are given below.

C.-H. Lin · F.-Y. Chang · S.-H. Chang

Mechanical and System Laboratories, Industrial Technology Research Institute, Hsinchu, Taiwan 310, Republic of China

H. Yang $(\bowtie) \cdot$ M.-T. Yen

Institute of Precision Engineering, National Chung Hsing University, Taichung, Taiwan 402, Republic of China

e-mail: hsiharng@nchu.edu.tw

The online version of the original article can be found under doi:10.1007/s00542-007-0521-z.

C.-H. Lin · F.-Y. Chang $\cdot$ S.-H. Chang

Mechanical and System Laboratories, Industrial Technology,

Research Institute, Hsinchu, Taiwan 310, Republic of China

H. Yang $(\varangle) \cdot$ M.-T. Yen

Institute of Precision Engineering,

National Chung Hsing University,

Taichung, Taiwan 402, Republic of China

e-mail: hsiharng@nchu.edu.tw 